\title{
REVISIÓN CRÍTICA DE LA IMPORTANCIA DE LA INNOVACIÓN EN EL CAMBIANTE AMBIENTE EMPRESARIAL
}

\section{CRITICALLY REVIEW THE IMPORTANCE OF INNOVATION IN THE CHANGING BUSINESS ENVIRONMENT}

Javier Benalcázar Ordóñez

freddy.benalcazar@ute.edu.ec

\section{Resumen}

La innovación crea cambios en el mundo empresarial, por lo que las empresas deben estar preparadas para los nuevos retos tecnológicos y tener la capacidad de dirigir a su capital humano y recursos financieros con éxito.

El objetivo de esta investigación es analizar los factores internos y externos donde desarrollan sus actividades las empresas, concepto de innovación y sus modelos, para que las empresas conozcan que la innovación es el camino para ser rentables en el mercado.

La investigación revisa importante literatura del método FODA, innovación, y ciclo de vida del producto para evaluar y tener una mejor comprensión de cómo las organizaciones pueden analizar y responder los cambios constantes y dinámicos de su entorno si quieren sobrevivir en el mercado.

También se realiza un análisis exploratorio del desarrollo de las actividades de la empresa Yanbal realizando una matriz FODA, y seguimiento en su volumen de ventas para poder determinar en forma general el desenvolvimiento de esta empresa desde que empezó sus operaciones en el Ecuador.

Palabras claves: Entorno empresarial, FODA, Innovación, Modelos de innovación, ciclo de vida del producto.

\begin{abstract}
Innovation creates changes in the business world, therefore companies must be prepared for new technological changes and have the ability to manage their human capital and financial resources successfully.

The objective of this research is to analyze the internal and external factors where companies carry out their activities, the concept of innovation and its models, with the purpose that companies know that innovation is the way to be profitable in the market.

The research reviews a literature of SWOT analysis, innovation, and product life cycle to assess and have a better understanding of how organizations can analyze and respond to constant and dynamic changes in their environment if they want to survive in the market.

An exploratory analysis is also carried out of the development of the Yanbal company's activities, both in evolution and sales, in order to determine in general terms the development of this company since it began its operations in Ecuador.
\end{abstract}

Keyword: Business environment, SWOT, Innovation, innovation models, products lifecycle. 


\section{Introducción}

Vivimos en un mundo global que es dinámico y cambia rápidamente es por eso que la capacidad de adaptarse y evolucionar con los cambios en el entorno empresarial se convierte en una verdadera necesidad para que las empresas no desaparezcan.

Una de las herramientas para que las empresas puedan identificar su ambiente interno y externo es la matriz FODA (SWOT).

Las empresas están viviendo diferentes cambios que traen consecuencias en sus estructuras y en su forma de tomar decisiones. Por lo tanto, es fundamental que identifiquen lo que está pasando en su entorno y así alcanzar un mejor posicionamiento en el mercado e incrementar sus ingresos. Las organizaciones deben estar preparadas a tomar decisiones que les permita ser competitivas y rentables en los nuevos retos del mundo empresarial. (Aguillo, 1999).

En lo referente a los productos y servicios que ofrecen las empresas pasan por diferentes etapas de vida en el mercado, desde su introducción hasta su desaparición, pasando por periodos de crecimiento y madurez; comparables con el ciclo de vida humano.

\section{2.-Entorno empresarial}

Las empresas se desarrollan con factores tanto internos como externos que determinaran su desarrollo y crecimiento. Las organizaciones no son entes aislados y por lo tanto tiene que tratar no solo con sus clientes sino con proveedores, y organizaciones relacionadas con su industria. Adicionalmente las empresas necesitan mejorar sus formas de producir y distribuir sus productos sin dejar nunca de analizar y acoplarse a los cambios económicos, tecnológicos (Innovación), políticos, culturales que afectan directamente con su crecimiento. (Gallardo, 2012)

En consecuencia, las empresas podrán determinar su entorno empresarial analizando sus factores controlables e internos que son: fortalezas y debilidades, así como sus factores no controlables y externos conocidos como oportunidades y amenazas (Porter, 1982)

Este análisis es conocido como método FODA (siglas en ingles SWOT) que ayuda a determinar la capacidad que tienen las empresas para hacer las cosas y a identificar lo que no pueden hacer. Además, este método ayuda a analizar las oportunidades y amenazas que tiene un mercado. Cuando el análisis FODA es desarrollado las empresas pueden determinar cómo pueden alcázar sus objetivos y como reducir riegos para alcanzar sus metas (Singh, 2010).

Por otro lado, (Ommani, 2011) señala que las empresas cuando realizan un análisis podrán contestar las siguientes preguntas:

1. Como usar sus fortalezas para tener ventaja competitiva sobre mi competencia

2. Como utilizar sus fortalezas para enfrentar con éxito los obstáculos

3. Cuál es la estrategia para vencer mis debilidades que me hace vulnerable con la competencia

4. Cuál es la estrategia para que mis debilidades no me lleven a fracasar en el mercado

Grafico 1: Entorno en una organización ANALIS FODA 

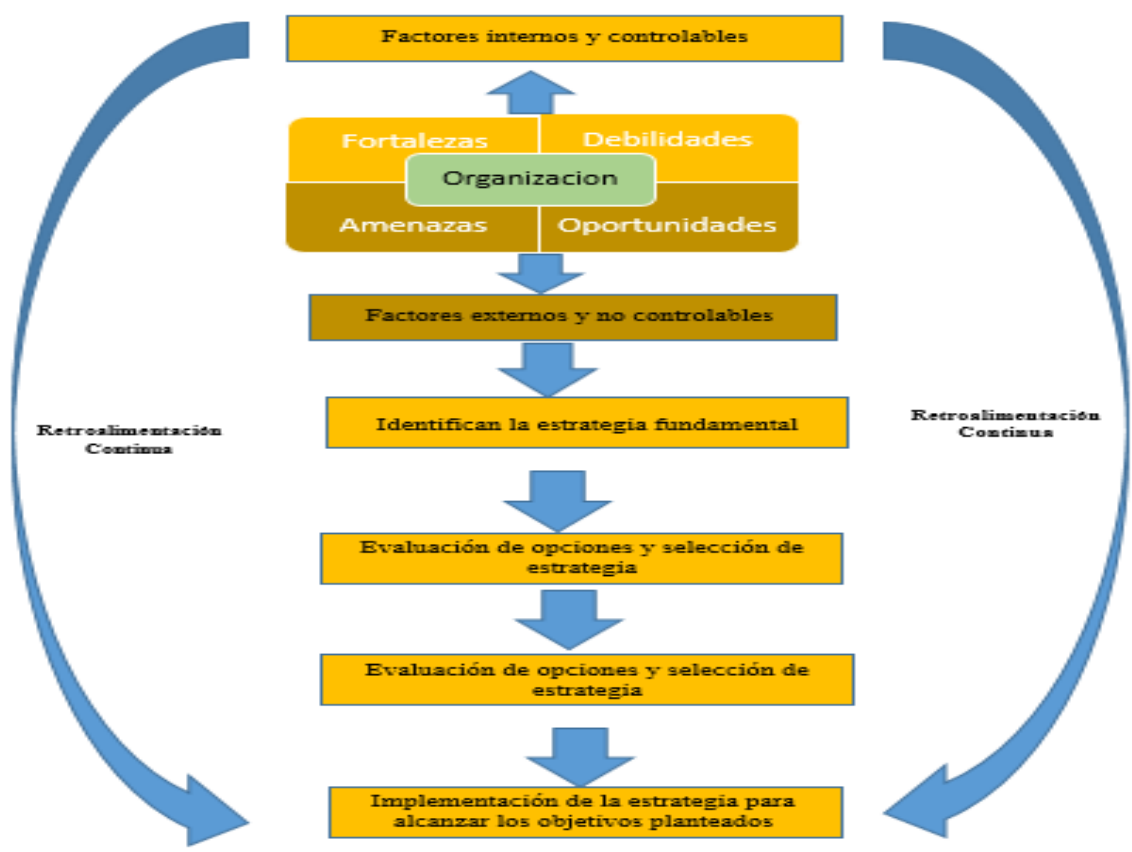

Fuente: Porter (1982); (Riston, 2008)

Las condiciones en donde se desarrollan las organizaciones son el factor determinante para el correcto crecimiento de las empresas, por lo tanto, el crecimiento es el resultado del ambiente sobre la industria seleccionada (Kangasharju, 2000)

Por otro lado, (Dobbs \& Hamilton, 2007), señalan que la variación en el tamaño de la demanda, el alcance del producto y la estabilidad de las empresas locales podrían afectar el crecimiento de las organizaciones. Durante periodos de alta demanda y madurez de las empresas se incrementa la posibilidad de crecimiento sostenido y sobrevivencia de las organizaciones.

En lo referente a la cadena de abastecimiento una variación de los costos y disponibilidad de materias prima, bienes manufacturados, maquinaria y otros insumos para la producción, podrían también estar relacionados con el desenvolvimiento de una organización en su industria (Dobbs \& Hamilton, 2007)

En el caso del Ecuador en lo concerniente a las importaciones tenemos a los Estados Unidos como un proveedor fuerte pero que están viendo como nuestro país está realizando compras significativas con los miembros de la Comunidad Andina, ALADI, Unión Europea y Asia, lo que ha permitido reducir costos de producción y en algunos casos tiempos de entrega. (Vasquez \& Saltos, 2013)

Adicionalmente, un factor determinante de donde se desarrollan las industrias es el nivel de competidores que éstas posean y los factores externos (amenazas y oportunidades del mercado) determinaran el crecimiento de las empresas. (Dobbs \& Hamilton, 2007)

Actualmente vivimos en la era de la globalización marcada por la relación entre países, reducción de barreras arancelarias, incremento de información (internet) y de network (red de contactos) originando que cambie el poder de negociación y de hacer negocios. Las empresas tienen la capacidad de alcanzar proveedores en todo el mundo y la posibilidad de vender su producto en todos los continentes. (Tse \& Soufani, 2003)

Las empresas saben que el mundo globalizando ha generado más que nunca antes una competencia voraz; por eso buscan su estrategia de crecimiento partiendo de las necesidades del cliente y finalizando con los recursos de las empresas (activos). (Slywotzky \& Morrison, 1997) 
Analizando la matriz FODA, tenemos que las empresas presentan las siguientes características:

Cuadro 1: ANALIS FODA

\begin{tabular}{|c|c|}
\hline FORTALEZAS & DEBILIDADES \\
\hline $\begin{array}{c}\text { Buen servicio al cliente } \\
\text { Manejo de gestión de calidad en: administración, } \\
\text { producción y distribución } \\
\text { Posicionamiento en el mercado } \\
\text { Capacitación constante para el personal } \\
\text { Poca rotación del personal }\end{array}$ & $\begin{array}{c}\text { Poco espacio para almacenamiento } \\
\text { Falta de control de ingresos y salidas de la } \\
\text { mercadería } \\
\text { Tiempo de cotización largo } \\
\text { Poca investigación y desarrollo Innovación) } \\
\text { Deficiente investigación de mercados } \\
\text { Falta de financiamiento } \\
\text { Poca infraestructura } \\
\text { Inadecuada política financiera y pagos } \\
\text { Incremento en el costo de los servicios básicos }\end{array}$ \\
\hline OPORTUNIDADES & AMENAZAS \\
\hline $\begin{array}{c}\text { Incremento de infraestructura mediantes inversión } \\
\text { publica } \\
\text { Políticas gubernamentales favorables para la } \\
\text { exportación } \\
\text { El producto posee características únicas y se ajusta } \\
\text { a las necesidades del mercado } \\
\begin{array}{c}\text { Suficiente empresas locales e internacionales que } \\
\text { produzcan insumos para la industria }\end{array} \\
\text { Tratados comerciales con los países tanto para } \\
\text { exportar como para importar }\end{array}$ & $\begin{array}{c}\text { La globalización permite que competidores fuertes } \\
\text { y más grandes de otros países puedan entrar al } \\
\text { mercado local } \\
\text { Disminución de pedidos por parte de clientes del } \\
\text { exterior } \\
\text { Cambio en la preferencia de los consumidores } \\
\text { Inestabilidad política y económica en el Ecuador } \\
\text { Rápido cambio tecnológico que vuelve obsoletos } \\
\text { los productos o menos atractivos para el } \\
\text { consumidor }\end{array}$ \\
\hline
\end{tabular}

Fuente: Flacso-Mipro (2011), (Hernandez, 2012)

Finalmente, según, (Cerveron \& Yibarra, 2016) el éxito de la innovación no radica solamente en tener acceso a nueva tecnología, ya que la verdadera ventaja que pueden tener las empresas es cuando tienen la capacidad de analizar su entorno para identificar amenazas y oportunidades lo que les permitirá establecer una correcta estrategia logrando éxito y adecuado manejo de sus recursos.

\section{Definición de innovación}

Las empresas en general están buscando innovar sus productos, servicios y procesos con el objetivo principal de ser o seguir siendo rentables; sin embargo, esto implica en muchos casos tiempo y dinero (Amit, R; Zott,C 2009).

La innovación puede ser conceptualizada desde diferentes enfoques, por lo que tenemos varios conceptos de innovación:

Joseph Shumpeter (en Goffin \& Mitchell 2005 p. 44) argumenta que la innovación implica no solo la creación de un producto sino que está también conectada a nuevos procesos de innovación en áreas como la fabricación y logística. Estos nuevos procesos, pueden cambiar las estructuras de costos de una industria. Él señala cinco tipos de innovación:

1. Producto nuevo o mejorado

2. Nuevas formas de producción

3. Creación de un nuevo mercado

4. Nuevas formas de adquirir materiales

5. El cambio de una industria genera nuevas formas de competencia

Adicionalmente, Trott (2002, p. 11) señalo que la innovación es el pilar para generar nuevas ideas, desarrollar tecnología, elaborar y distribuir (logística) los productos en una forma cada vez mejor y así obtener cambios que darán éxito a nuestra organización. 


\subsection{Modelos de innovación}

Las innovaciones han ido cambiando en el tiempo, es así que, con el paso de la historia, hemos tenido diferentes modelos de innovación. En esta investigación se analiza las características y diferencias de 4 modelos.

Cuadro 2. Diferencias entre el modelo lineal y el modelo interactivo

MODELO LINEAL

Es cuando se tiene una secuencia de una etapa a otra. Este modelo tiene diferentes etapas las cuales son:

- Investigación básica (Fuente inicial de la innovación)

- Diseño e ingeniería,

- Producción, y;

- Mercadeo

Tecnología Push.- La investigación básica y la tecnología crea un nuevo producto

Market Pull. - Este sufre una variante en relación al modelo inicial lineal, ya que aunque conserva el carácter secuencial y lineal indica que el mercado es quien impulsa la investigación y el desarrollo para crear un nuevo producto. Las etapas aquí son:

- Factores de la demanda del mercado (Fuente inicial de la innovación),

- Diseño e Ingeniería,

- Producción, y;

- Ventas (Marketing)

Las innovación nacen de los conocimientos científicos

\section{MODELO INTERACTIVO}

Enlaza y se retroalimenta de los conocimientos científicos, tecnológicos y del mercado. La innovación se genera simultáneamente de:

- Conocimientos previos

- Tecnología disponible

- Demanda del mercado

- Nuevos diseños

- Diseños en pruebas

- Rediseños (retroalimentación)

- Distribución del producto

La innovación no es un proceso aislado todo el conjunto crea un nuevo producto.

La creación no es lineal

La organización es quien tiene un papel fundamental en el proceso innovador.

El nuevo producto se crea utilizando:

- Recursos de la organización,

- Requisitos del mercado, y;

- Tecnología existente.

Acepta que la innovación puede nacer de la propia experiencia y de los conocimientos científicos

Fuente: Trott (2002), Retana, L, Chryssopoulos R, Esquivel, Ronald (2015), Gonzalez, C (s.f)

Cuadro 3. Diferencias y características entre innovación abierta e innovación cerrada

\section{INNOVACIÓN ABIERTA}

- La innovación nace de una acción colectiva.

- Involucra a socios externos, como clientes, proveedores, universidades, gobiernos.

- Las empresas no necesitan contratar a las personas con más capacidad

\section{INNOVACIÓN CERRADA}

- Acción individual

- Las Universidades no poseen importancia

- Las empresas deben contratar a las personas más capaces para desarrollar un nuevo producto 


\begin{tabular}{|c|c|}
\hline $\begin{array}{l}\text { Usa recursos de innovación internos y } \\
\text { externos para asegurar dar el mejor valor al } \\
\text { producto, servicio o mercadeo } \\
\text { Para ser competitivos es necesario aplicar el } \\
\text { mejor modelo de negocios en lugar de entrar }\end{array}$ & $\begin{array}{l}\text { Se lidera la industria invirtiendo en } \\
\text { investigación y desarrollo (I\&D) dentro de la } \\
\text { organización, logrando generar nuevas ideas } \\
\text { desde el interior de la compañía y así obtener } \\
\text { ventaja sobre la competencia }\end{array}$ \\
\hline \multirow{3}{*}{$\begin{array}{l}\text { Reduce costos y riesgos en el momento de } \\
\text { diversificar tecnología y/o mercados } \\
\text { Comparte su desarrollo e investigación con } \\
\text { sus socios comerciales externos. }\end{array}$} & $\begin{array}{l}\text { La organización para obtener rentabilidad debe } \\
\text { utilizar solo sus capacidades }\end{array}$ \\
\hline & $\begin{array}{l}\text { Asume todos los costos y riesgos de los } \\
\text { proyectos }\end{array}$ \\
\hline & $\begin{array}{l}\text { La compañía descubre, desarrolla y } \\
\text { comercializa por si misma. }\end{array}$ \\
\hline $\begin{array}{l}\text { Implica un intercambio de conocimiento } \\
\text { Toma las mejores ideas internas y externas }\end{array}$ & Cubre el conocimiento \\
\hline $\begin{array}{l}\text { Las firmas más grandes son más abiertas a } \\
\text { compartir su innovación que los Pymes } \\
\text { (pequeñas y medianas industrias) }\end{array}$ & $\begin{array}{l}\text { Ser el primero en el mercado se origina } \\
\text { cuando el descubrimiento de las } \\
\text { investigaciones se originan dentro de la } \\
\text { misma compañía }\end{array}$ \\
\hline $\begin{array}{c}\text { Nacen las alianzas, joint ventures, joint } \\
\text { development, adquisiciones, contratos de } \\
\text { investigación y desarrollo, licencias } \\
\text { Desarrollo dinámico de propiedad intelectual } \\
\text { (IP) }\end{array}$ & $\begin{array}{c}\text { Hay que utilizar leyes restrictivas y políticas } \\
\text { de propiedad intelectual (IP) para prevenir } \\
\text { que la competencia obtenga ganancias de las } \\
\text { ideas y tecnología desarrolladas por la } \\
\text { empresa }\end{array}$ \\
\hline
\end{tabular}

Fuente: (www.oecd.org, 2008), (Gemunden, H, et al 2011)

En resumen podemos decir que la innovación abierta aporta nueva riqueza para las organizaciones. Por ejemplo, durante una gran recesión en Argentina, Repsol YPF en sociedad con Volkswagen lanzó un automóvil con gas natural comprimido. El automóvil necesitaba usar gas en lugar de gasolina y esto le permitió al cliente ahorrar dinero. El nuevo motor fue difícil de copiar rápidamente. Adicionalmente, Volkswagen ofreció servicios completos de instalación y registro para el gobierno. Esta alianza cumplió con las necesidades del cliente y permitió que las empresas se posicionen fuertemente en el mercado. (Goffin \& Mitchel 2005).

\section{Ciclo de vida de los productos}

El escenario de ciclo de vida de los productos se ha visto modificado por la constante innovación y la globalización de mercados. El análisis del ciclo de vida de los productos ayuda a diseñar las estrategias de comercialización y mercado durante todo el proceso, las cuales permiten obtener a las empresas ventas y beneficios. (Godas, 2006).

Según Glessner, L, Gillis, D (2012) señala que el ciclo de vida de los productos abarca las diferentes etapas que los productos y servicios experimentan en su tiempo de vida en el mercado. Cada etapa tiene diferentes características y estrategias de negocios. La correcta aplicación de las estrategias permitirá a las empresas mantener rentabilidad y asegurarse una saludable mezcla de líneas de productos dentro del continuo ciclo de vida en que está envuelto un producto. 
La duración de cada fase puede variar de pocos meses a años, eso dependerá de la estructura de la industria, los competidores, políticas gubernamentales, la innovación, tecnología, la política de precios y las estrategias de la empresa (www.managersmagazine.com)

Levitt (1965) describió el primer modelo del ciclo de vida del producto señalando que existen 4 etapas, las cuales son:

Gráfico 2. Etapas del ciclo de vida del producto

DESARROLLO

El producto se introduce al mercado

Lento crecimiento

Ventas bajas

Gastos altos en promoción

Pocos o ningún tipo de competidores

Alta inversión técnica y comercial Enfrentamiento de posibles barreras $\mathrm{d}$ entrada al mercado

\section{CRECIMIENTO}

El producto comienza a posicionarse en la mente del consumidor

Se comienza a expandir el mercado Se incrementan las ventas

Muchos competidores entran al mercado Mejora el proceso de producción y existe nuevas versiones del producto

Altos costos y reinversión de beneficios

\section{Etapa II \\ Etapa III \\ Fuente: Levitt (1965) y Godas (2006) \\ Elaborado por: Javier Benalcázar}

Se alcanza el mayor posicionamiento en el mercado

La demanda crece

ligeramente debido a

la renovación del producto y crecimiento poblacional

Incremento de líneas del producto Mejora en la fabricación y reducción de costos de producción, distribución Número de

competidores alto

\section{DECLIVE}

Los consumidores empiezan a optar por otro tipo de productos La demanda empieza a decrecer

Los nuevos productos innovadores empiezan

a tener mayor aceptación por el consumidor (desplazan al antiguo producto) La distribución es selectiva

\section{Caso de estudio: Yanbal}

En el Ecuador una de las empresas que se ha convertido en un referente en la industria cosmética es Yanbal Ecuador. Esta organización fue fundada en el Perú en el año 1967. Su línea de productos abarca cosméticos, fragancia, cuidado personal y bisutería.

El presente trabajo presenta una investigación exploratoria de la empresa Yanbal realizando un análisis FODA y un análisis del desarrollo de sus actividades en el Ecuador. 


\begin{tabular}{|c|c|}
\hline $\begin{array}{c}\text { Ayuda al crecimiento profesional de consultores y } \\
\text { directores } \\
\text { Capacita a su personal de ventas constantemente } \\
\text { Buen servicio de post-venta } \\
\text { Consultores y directores conocen el producto } \\
\text { ofreciendo lo que necesitan los clientes } \\
\text { Experiencia en el mercado } \\
\text { Ofrecen asesoría personalizada } \\
\text { Excelente presentación de su catalogo } \\
\text { Posicionamiento en la mente del ecuatoriano } \\
\text { Aplicación de tecnología para mejor su sistema de } \\
\text { producción y logística } \\
\text { Uso de la tecnología para innovar sus productos }\end{array}$ & $\begin{array}{c}\text { Falta de dinero para empezar el negocio en Yanbal } \\
\text { Falta de disponibilidad de ciertos productos } \\
\text { El crédito otorgado es mayor a la capacidad de } \\
\text { pago } \\
\text { Falta de tiempo para asistir a los cursos de } \\
\text { actualización } \\
\text { Productos con precios altos } \\
\text { Poca agresividad para obtener clientes }\end{array}$ \\
\hline OPORTUNIDADES & AMENAZAS \\
\hline $\begin{array}{c}\text { Mejora el estilo de vida de las personas que } \\
\text { empiezan en Yanbal (carrera profesional) } \\
\text { Existe buena rentabilidad de ganancia en cada } \\
\text { producto Yambal } \\
\text { El alza de desempleo en el Ecuador colabora a que } \\
\text { más personas quieran ser consultoras } \\
\text { Amplia variedad de productos de belleza } \\
\text { Implementación de avances tecnológicos } \\
\text { (Innovación) }\end{array}$ & $\begin{array}{c}\text { Fuerte competencia de empresas que venden } \\
\text { productos de belleza en forma directa } \\
\text { Precios altos referente a sus competencia } \\
\text { El desempleo origina que las personas bajen su } \\
\text { poder adquisitivo de productos de belleza } \\
\text { Las ventas por catálogo no permiten conocer } \\
100 \% \text { el producto } \\
\text { Pago de derecho arancelario del } 20 \% \text { que } \\
\text { incrementa el valor de sus productos } \\
\text { Incremento de arancel para las materias primas } \\
\text { La competencia utiliza la televisión para llegar a } \\
\text { mas consumidores }\end{array}$ \\
\hline
\end{tabular}

Fuente: Yanbal (2018) y Modelos de Gestión Empresarial (2016) Elaborado por: Javier Benalcázar

Para determinar el desarrollo de las actividades de Yanbal en Ecuador la investigación analizo los eventos en orden cronológico que tuvo esta empresa desde 1997. (Cuadro 5).

Cuadro 5. Operación de Yanbal en Ecuador desde 1997

\begin{tabular}{|c|c|c|}
\hline Años & Eventos & $\begin{array}{l}\text { Ingresos en } \\
\text { millones de } \\
\text { dólares } \\
\text { (Ventas) }\end{array}$ \\
\hline 1977 & $\begin{array}{c}\text { Yanbal ingresa al mercado ecuatoriano. Su primer centro de } \\
\text { distribución fue en Guayaquil }\end{array}$ & \\
\hline 1989 & Las operaciones centrales se trasladan a Quito & \\
\hline 1990 & Construcción de la planta de cosméticos en el Ecuador & \\
\hline 1996 & $\begin{array}{l}\text { La innovación empieza con la implementación del sistema } \\
\text { MAPICS }\end{array}$ & \\
\hline 1999 & $\begin{array}{c}\text { El país sufrió una gran crisis económica y la estrategia de Yanbal } \\
\text { fue "cuidar a las personas" (consultoras, directoras, empleado y } \\
\text { proveedores) }\end{array}$ & \\
\hline 2001 & $\begin{array}{l}\text { Se incrementa la producción gracias a la inauguración de la planta } \\
\text { de producción al pasar la planta a las instalaciones de Righetti }\end{array}$ & \\
\hline 2003 & Se introduce un sistema de pedidos y pagos vía celular & 105,3 \\
\hline
\end{tabular}




\begin{tabular}{|c|c|c|}
\hline Años & Eventos & $\begin{array}{l}\text { Ingresos en } \\
\text { millones de } \\
\text { dólares } \\
\text { (Ventas) }\end{array}$ \\
\hline 2004 & $\begin{array}{l}\text { 1er lugar Sector no financiero Jabones, cosméticos y artículos de } \\
\text { limpieza. Suben las ventas en un } 28,2 \% \text { en relación al año pasado }\end{array}$ & 135 \\
\hline 2005 & II lugar con el mejor lugar para trabajar & \\
\hline 2006 & $\begin{array}{c}\text { Gana el Ekos de Oro como 1er lugar Sector no financiero Jabones, } \\
\text { cosméticos y artículos de limpieza }\end{array}$ & \\
\hline \multirow{2}{*}{2009} & Gana 5to lugar - Great Place to Work & \\
\hline & Se construye el nuevo centro de distribución en Aloag & \\
\hline \multirow{2}{*}{2010} & Se invierte en el sistema ERP llamado SAP & \multirow{2}{*}{171,200} \\
\hline & Cuenta con 700 colaboradores & \\
\hline \multirow{5}{*}{2011} & $\begin{array}{c}\text { Yambal posee } 12 \text { localidades en ciudades como Quito, Guayaquil, } \\
\text { Cuenca, Machala, Manta, Santo Domingo, Ambato, Loja, } \\
\text { Esmeraldas. }\end{array}$ & \multirow{5}{*}{178,000} \\
\hline & Gana el 1er lugar - Ekos de Oro - 2011 & \\
\hline & $\begin{array}{c}\text { Del año } 2010 \text { al } 2011 \text { consiguió un ahorro de USD } 50000 \text { gracias a } \\
\text { su política del medio ambiente }\end{array}$ & \\
\hline & 1er lugar Sector no financiero Productos de belleza & \\
\hline & Se tiene 688 colaboradores & \\
\hline \multirow{3}{*}{2012} & $\begin{array}{c}\text { Realiza inversiones en proyectos de desarrollo sostenible para } \\
\text { optimizar procesos reduciendo gastos financieros y no financieros }\end{array}$ & \multirow{3}{*}{195,707} \\
\hline & $\begin{array}{c}\text { Incorpora un solo sistema Integrado de Gestión SIG para optimizar } \\
\text { recursos }\end{array}$ & \\
\hline & $\begin{array}{l}\text { 8vo lugar Empresas con mejor calidad de servicio Libre Compra y } \\
\text { 4to lugar Empresas con mayor capital de marca Capital de marca }\end{array}$ & \\
\hline \multirow[t]{2}{*}{2013} & $\begin{array}{c}\text { Sigue fortaleciendo su responsabilidad social brindando mejores } \\
\text { condiciones para la educación y apoyo a niños y niñas, y jóvenes } \\
\text { con capacidades especiales en el Ecuador. Adicionalmente trabajo } \\
\text { con la Corporación Ecuatoriana de Cooperación e Inclusión de las } \\
\text { mujeres (CECIM) }\end{array}$ & \multirow[t]{2}{*}{206,628} \\
\hline & Gana el 9vo lugar como marca más recordada & \\
\hline 2014 & $\begin{array}{l}\text { Se coloca en el ranking } 18 \text { de empresas con mejor reputación en el } \\
\text { Ecuador. Finaliza su vinculación con los proyectos sociales. }\end{array}$ & 210,037 \\
\hline 2015 & $\begin{array}{l}\text { En el Ecuador ocupa el puesto } 28 \text { de empresas con mejor ranking } \\
\text { y una participación en el mercado del } 27,9 \%\end{array}$ & 206,537 \\
\hline 2016 & Las ventas sufren una caída importante & 163,333 \\
\hline 2017 & Las ventas siguen decayendo & 130 \\
\hline
\end{tabular}

Fuente: Merco (2016); Revista Ekos, varias publicaciones; Revista lideres 2015, Mayo 2015; Informe de sostenibilidad Corporativa Yanbal Ecuador 2010-2011; Martínez, M (2006)

Elaborado por: Javier Benalcázar

Adicionalmente en este estudio se detalla los niveles de ventas en forma integral de Yanbal desde el año 2003 hasta el 2016, con el objetivo de analizar como evolucionaron las ventas totales de los productos de esta organización.

Cuadro 6. Comportamiento de ventas de Yanbal Ecuador

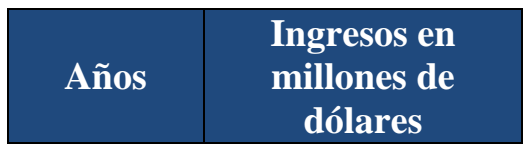




\begin{tabular}{|c|c|}
\hline & (VENTAS) \\
\hline 2003 & 105,3 \\
\hline 2004 & 135 \\
\hline 2010 & 171,200 \\
\hline 2011 & 178,000 \\
\hline 2012 & 195,707 \\
\hline 2013 & 206,628 \\
\hline 2014 & 210,037 \\
\hline 2015 & 206,537 \\
\hline 2016 & 163,333 \\
\hline 2017 & 130 \\
\hline
\end{tabular}

Fuente: Merco (2016); Martínez M (2006); (Ekosnegocios.com); El Universo (2018)

Elaborado por: Javier Benalcázar

El cuadro indica que Yanbal incrementa sus ventas desde el año 2003 al 2014 donde alcanza los 210,037 millones en ventas. En los años siguientes sus ventas caen hasta alcanzar los 130 millones en el 2017.

Yanbal, desde el año 2015 ha empezado a reducir sus ventas por lo que surge la pregunta: ¿A que se debe la reducción de ventas? ¿Está la empresa afectada por la situación económica que vive el Ecuador?

Gráfico 4. Ingreso de las ventas de Yanbal en millones de dólares

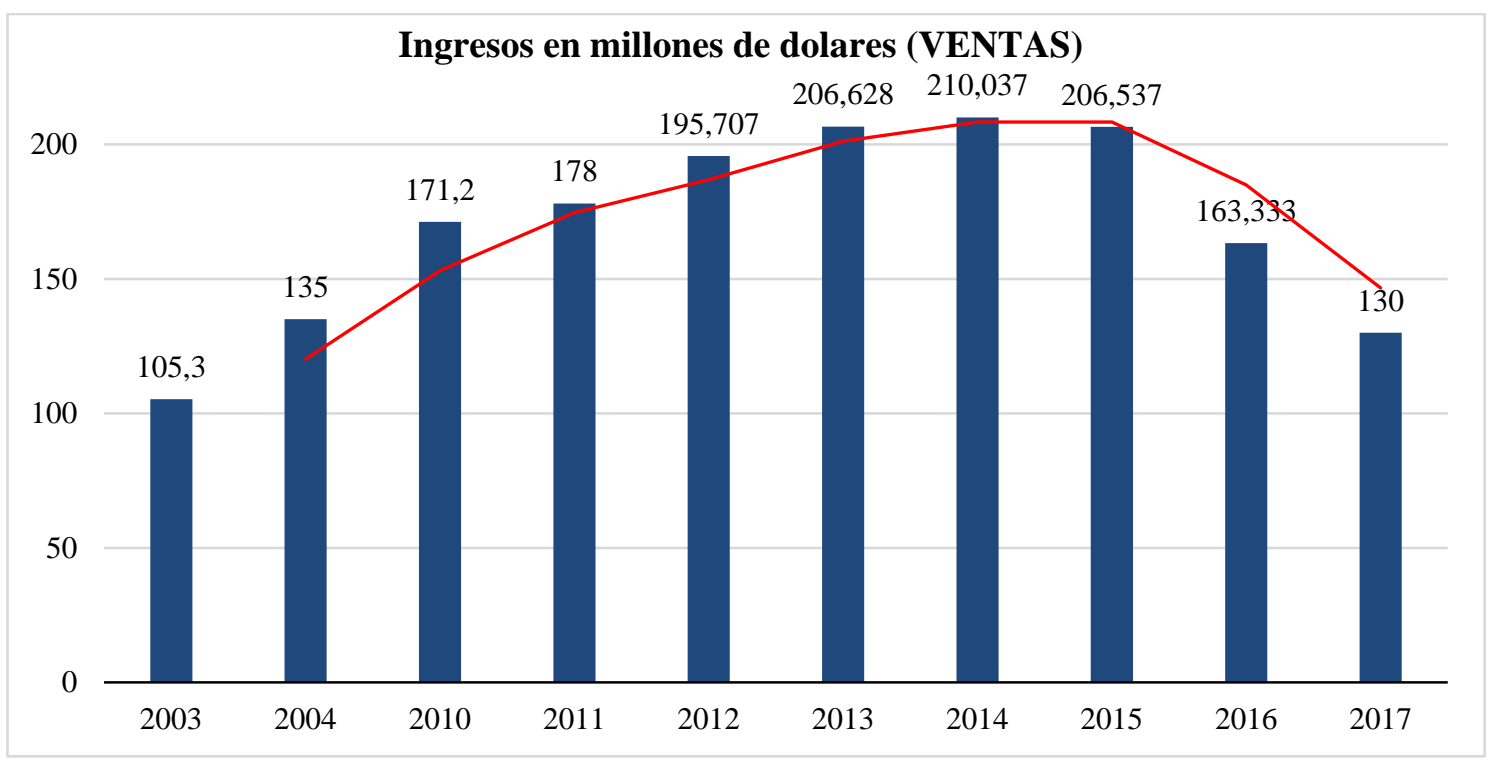

Fuente: Merco (2016); Martínez M (2006); (Ekosnegocios.com); (UNIVERSO, 2018)

Elaborado por: Javier Benalcázar

\section{Análisis:}

Como podemos observar durante estos años Yanbal, ha tenido el acierto de ver las oportunidades y amenazas en el mercado ecuatoriano. Esta empresa que motiva a sus consultores y staff de ventas no solo ha incrementado su línea de productos y ha mantenido su 
calidad sino que ha realizado importantes inversiones en áreas de producción y distribución. Yanbal aparte de hacer inversiones en nuevos centros de distribución identifico e implemento la nueva tecnología para mejorar sus procesos para mejorar costo y ser más rentables, es por eso que introduce un sistema de pedidos y pago vía celular, implementa el sistema ERP SAP (sistema de planificación de recursos empresariales), el cual es utilizado por grandes multinacionales como Siemens.

Adicionalmente esta organización entendió que el mundo empieza a no solo ver la calidad del producto sino también es más consiente por el medio ambiente y la seguridad de los trabajadores es así que incorpora un nuevos sistema de gestión (SIG) el mismo que está conformado por calidad, medio ambiente y prevención de riesgos laborales. Lo que le ha llevado a colocarse y mantenerse como una de las mejores empresas a trabajar en el Ecuador. En 2014 la organización alcanzo el ranking 18 como empresa de mejor reputación en el país.

Finalmente Yanbal ha ganado diferentes reconocimientos como el Ekos de Oro, calidad de servicio libre compra, marca recordada, entre otras. Es por eso que podemos ver que Yanbal entendió la fuerte competencia que se tiene en la industria de la belleza, la necesidad de mantener calidad, de diversificar productos, de tecnificarse con las nuevas innovaciones, de estar en línea con las nuevas demandas de medio ambiente y protección a los trabajadores que le han permitido seguir con éxito sus operaciones en el Ecuador y que con seguridad llego al país para quedarse y ser un ejemplo de organización a seguir.

\section{Conclusión}

Las organizaciones deben adaptarse a sus nuevos entornos generados en muchas ocasiones por la innovación lo antes posibles, para así poder tomar decisiones y estrategias acertadas que les permitan mantenerse en el mercado y seguir siendo rentables.

Las organizaciones desarrollan sus actividades en medio de factores internos como externos, los cuales determinan el éxito y posicionamiento en el mercado.

Las empresas podrán identificar su entorno utilizando la matriz FODA la misma que ayuda a identificar fortaleza y debilidades (factores controlables) que poseen las empresas, así como las oportunidades y amenazas que existe en el mercado (factores no controlables). Cuando las empresas determinan su entorno (factores externos e internos) tendrá la capacidad de saber qué es lo que pueden realizar, donde están sus limitaciones.

como aprovecharse las oportunidades que existen en el mercado y como enfrentar de mejor manera las amenazas que tendrán que afrontar.

Las condiciones donde las empresas realicen sus actividades serán el factor fundamental para lograr sus objetivos, es así que el crecimiento será posible gracias al ambiente donde ellas se encuentren. Los factores como recursos financieros, acuerdos comerciales, cantidad de competidores, disponibilidad de materia prima, insumos, maquinaria, o bienes finales marcaran el camino para el desarrollo de las organizaciones.

La capacidad de adaptarse a los nuevos cambios permitirá a las empresas manejar de mejor manera sus recursos y establecer estrategias que les lleven a tener una ventaja sobre sus competidores.

La innovación no se centra solamente en la creación de nuevos productos sino también abarca procesos tales como: fabricación, producción, ensamble, envase y empaquetamiento y logística.

Los modelos de innovación han servido para llenar las necesidades y demanda de los consumidores pero también para generar riquezas para las organizaciones. 
La globalización y la innovación han modificado el ciclo de vida de los productos. Por lo que es necesario que las empresas siempre hagan un análisis de su entorno y así poder aplicar la mejor estrategia posible.

Las empresas que entiendan la importancia de entender su entorno empresarial y del papel que juega la innovación son las que generaran riqueza, serán más productivas, alcanzaran ventaja competitiva, ganaran reconocimiento, se posicionaran en la mente de los consumidores y serán un ejemplo a seguir en el cambiante mundo de los negocios.

\section{Bibliografía:}

Aguillo, C (1999). Cambios significativos en el mundo empresarial, Economía Industrial N0. 330. Obtenido de: http://www.minetad.gob.es/Publicaciones/Publicacionesperiodicas/EconomiaIndustrial/

RevistaEconomiaIndustrial/330/05cagu.pdf

Amit R; Zott, C (2010). Business Model Innovation: Creating Value in Times of Change Working Paper 870, IESE Business School, University of Navarra, 17 pages

Bárbara, R (2011) Panorámica Actual de la Cultura Ecuatoriana, Alpamanda Editorial

Cerveron. V; Yibarra J. (2016). La innovacion empresarial en Ontinyent y su entorno. Alicante: Universidad de Alicante.

Dobbs, \& Hamilton. (2007). Small business growth: recent evidence and new directions. International Journal of Entrepreneurial Behaviour \&Research, 13(5), 296-322.

Ekos. (2012). Yanbal, La Oportunidad "El producto Estrella" 2 de Octubre 2012 Obtenido de: http://www.ekosnegocios.com/negocios/verArticuloContenido.aspx?idArt=969

Ekos. (s/f). Guía de negocios 13 de noviembre de 2017 Obtenido de: http://www.ekosnegocios.com/empresas/empresas.aspx?idE=53

Flacso - Mipro (2011). Elaboración de Autopartes para el sector automotor . FLACSO MIPRO . Quito : Centro de investigaciones Economicas y de la Micro, pequeña y mediana Empresa. Recuperado el 07 de mayo de 2018, de https://www.flacso.edu.ec/portal/pnTemp/PageMaster/07xhc02kdn0i8zlp9o7iz17oh19g cv.pdf

Gallardo, H. (2012). Administracion estrategica de la Vision a la Ejecucion. Mexico:

Alfaomega Grupo Editor, S.A. de C.V. Mexico.

Knight, G. (2000). Entrepreneurship and Marketing Strategy. Journal of International Marketing, 8(2), 12-32

Glessner, L, Gillis, D (2012). The Knowledge Product Lifecycle and the Strategic Dashboard

Godas, L (2006).El Ciclo de vida del Producto, Offarm, Vol 25, Num 8 Obtenido: http://apps.elsevier.es/watermark/ctl_servlet?_f=10\&pident_articulo=13094134\&pident_usuario $=0 \&$ pident_revista $=4 \&$ fichero $=4 \mathrm{v} 25 \mathrm{n} 08 \mathrm{a} 13094134 \mathrm{pdf001}$.pdf $\&$ ty $=151 \&$ accion $=$ L \&origen $=$ do ymafarma\&web=www.doymafarma.com\&lan=es 
Gemunden, $\mathrm{H}$ et al (2011), Open and Close Innovation, Different Cultures for Different

Gonzales, C (s.f ) El proceso de la innovación . Universidad Nacional de la Plata Obtenido de: http://secyt.presi.unlp.edu.ar/cyt_htm/jornadacyt/conrado_gonzalez/21_innovacion.pdf

Goffin, K \& Mitchell, R. 2005, Innovation Management, Strategy and Implementation using the Pentathlon Framework, Creative Print \& Design, Great Britain.

Hernandez, J. (2012). Administarcion Estrategica de la Vision a la Ejecucion. Mexico: Alfaomega Grupo Editor, S.A de C.V.

Kangasharju, A. (2000). Grow of the smallest: determinants of small firm growth during strong macroeconomic fluctuations. International Small Business, 19(1), 28-43.

Levitt, T. (1965) Exploit the Product Life Cycle. Harvard Business Review, 43, 81-94.

Managermagazine.com (2013). El ciclo de vida del producto en marketing, 21 de diciembre 2013. Obtenido de: http://managersmagazine.com/index.php/2013/12/el-ciclo-de-vida-delproducto-en-marketing/

Maldonado, P (2015) Un Protagonista de los cosméticos Revista lideres 10 de Mayo de 2015 obtenido de: http://www.revistalideres.ec/lideres/cosmeticos-ecuador-empresas-yanbal.html

Martinez, M (2006). Mercado tentador: Venta de cosméticos. Microeconomía, Obtenido de: http://www.monografias.com/trabajos34/venta-cosmeticos/venta-cosmeticos.shtml

Merco. (2016). Merco Empresa 12 de noviembre de 2017, Obtenido de: http://merco.info/ec/ranking-merco-empresas

Modelos de Gestión Empresarial (9-Enero 2016), Yanbal Cadena de Valor, fecha de recuperación: 09/05/2018 del:

http://modelosdegestionempresarialcasos.blogspot.com/2016/01/yanbal-cadena-de-valor_9.html

50Minutos.es (2016). El ciclo de vida del producto. Optimice el desarrollo de sus productos en el mercado. Editorial. 50Minutos.es

OECD (2008). Open Innovation in Global Networks Obtenido de: https://www.oecd.org/innovation/inno/41446671.pdf

Ommani, A. (2011). Strengths, weaknesses, opportunities and threats (SWOT) analysis for farming system businesses management: Case of wheat farmers of Shadervan District, Shoushtar Township, Iran. African Journal of Business Management, 5(22), 9448 9454. Recuperado el domingo de 05 de 2018, de http://www.academicjournals.org/article/article1380639652_Ommani.pdf

Porter, M. (1982). Estrategia Competitiva: Estrategias para el analisis de los sectores industriales y de la competencia. Mexico : CECSA.

Retana, L, Chryssopoulos, R, Esquivel, Ronald, (2015). Evolución de los modelos en los procesos de innovación, una revisión de la literatura. Tecnología en Marcha. Vol. 29, No 1, Enero - Marzo. Pag 108-117 
Sanchez, E (2015), Ciclo de Vida de Producto. Modelos y Utilidad para el Marketing.

Singh, N. (2010). SWOT Analysis A Useful Tool For Community Vision A concept paper of central Himalayan village. 2(9), 16-18. Obtenido de http://www.sciencepub.net/researcher/research0209/

Slywotzky, A., Morrison, D. (1997). The Profit Zone: How Strartegic Business Design will Lead you to Tomorrow's Profits. Chichester: John Wiley \&Sons .

Stonehouse et.al. (2004). Global And Transnational Business, John Wiley\&Sons, Ltd, England.

Trot, P. (2002). Innovation Management and New Product Development, Person Education Limited, England.

Tse, T., \& Soufani, K. (2003). Business Strategies for small firms in the new economy. Journal of Small Business and Enterprise Development, 306-320.

Yanbal (2011), Informe de sostenibilidad Corporativa Yanbal Ecuador 2010-2011 obtenido de: http://info01.yanbal.com/ecuador/informe/informe-ec-2013.pdf

Yanbal (2018), Fecha de recuperacion de informacion: 09/05/2018 del: http://mundoyabal.blogspot.com/p/matriz-dofa.html

UNIVERSO. Obtenido de https://www.eluniverso.com/noticias/2018/03/21/nota/6676651/diafragancias-medio-mas-ventas obtenido: 10 de Mayo 2018

Vasquez, L., \& Saltos. (2013). Ecuador y su Realidad. Quito: Fundacion de Investigacion y Promocion Social ""Jose Peralta". 